\title{
DNA methylation, through DNMT1, has an essential role in the development of gastrointestinal smooth muscle cells and disease
}

Brian G. Jorgensen ${ }^{1}$, Robyn M. Berent ${ }^{1}$, Se Eun Ha ${ }^{1}$, Kazuhide Horiguchi ${ }^{2}$, Kent C. Sasse ${ }^{3}$, Laren S. Becker ${ }^{4}$ and Seungil $\mathrm{Ro}^{1}$

\begin{abstract}
DNA methylation is a key epigenetic modification that can regulate gene expression. Genomic DNA hypomethylation is commonly found in many gastrointestinal (Gl) diseases. Dysregulated gene expression in Gl smooth muscle cells (GlSMCs) can lead to motility disorders. However, the consequences of genomic DNA hypomethylation within GI-SMCS are still elusive. Utilizing a Cre-lox murine model, we have generated SMC-restricted DNA methyltransferase 1 (Dnmt 1) knockout (KO) mice and analyzed the effects of Dnmt1 deficiency. Dnmt1-KO pups are born smaller than their wildtype littermates, have shortened GI tracts, and lose peristaltic movement due to loss of the tunica muscularis in their intestine, causing massive intestinal dilation, and death around postnatal day 21. Within smooth muscle tissue, significant CpG hypomethylation occurs across the genome at promoters, introns, and exons. Additionally, there is a marked loss of differentiated SMC markers (Srf, Myh11, miR-133, miR-143/145), an increase in pro-apoptotic markers (Nr4a1, Gadd45g), loss of cellular connectivity, and an accumulation of coated vesicles within SMC. Interestingly, we observed consistent abnormal expression patterns of enzymes involved in DNA methylation between both Dnmt1-KO mice and diseased human Gl tissue. These data demonstrate that DNA hypomethylation in embryonic SMC, via congenital Dnmt1 deficiency, contributes to massive dysregulation of gene expression and is lethal to GI-SMC. These results suggest that Dnmt1 has a necessary role in the embryonic, primary development process of SMC with consistent patterns being found in human Gl diseased tissue.
\end{abstract}

\section{Introduction}

The aberrant growth, or loss, of smooth muscle cells (SMCs) is a common symptom found in burdensome gastrointestinal (GI) diseases such as chronic intestinal pseudo-obstruction ${ }^{1}$ and megacystismicrocolon-intestinal hypoperistalsis syndrome ${ }^{2}$ as well

Correspondence: Seungil Ro (sro@med.unr.edu)

${ }^{1}$ Department of Physiology and Cell Biology, University of Nevada School of Medicine, Reno, NV 89557, USA

${ }^{2}$ Department of Morphological and Physiological Sciences, University of Fukui, Fukui 910-8507, Japan

Full list of author information is available at the end of the article

These authors contributed equally: Brian G. Jorgensen, Robyn M. Berent, Se Eun Ha.

Edited by D. Heery 
sites can be found clustered together in regions entitled CpG islands that are often found in close proximity to, or within, transcription start sites (TSS) as well as the promoters of housekeeping and/or tissue-specific genes ${ }^{9}$. The addition of a methyl group to cytosine at its fifth carbon, creating 5-methylcytosine (5-mc), by DNA methyltransferases (DNMT1, DNMT3A, DNMT3B) can cause repression of associated genes as this mark, at and around the TSS, has been linked to transcriptionally repressed chromatin that denies access for transcription factors, such as serum response factor $(\mathrm{SRF})^{10,11}$. Thus, as SRF is a master regulator of contractile genes in $\mathrm{SMC}^{12}$, the transcription of many contractile smooth muscle genes (Myh11, Acta2) ${ }^{13,14}$ and microRNA (miRNAs; mir-133, mir-143/ $145)^{15,16}$ is dependent on the binding of SRF to CArG (CC $\left.[\mathrm{A} / \mathrm{T}]_{6} \mathrm{GG}\right)$ boxes at these loci ${ }^{17,18}$. CArG boxes are highly conserved, have high $\mathrm{CpG}$ content in the region bound by SRF, are commonly found within promoter regions/close to TSS, and are recognized specifically by $\mathrm{SRF}^{17}$. Additionally, high CpG content sites, such as those around CArG boxes, are also well-established targets for cytosine methylation by DNA methyltransferases ${ }^{19,20}$. While several studies have given valuable insight into the role of DNA methylation in SMC, many of them similarly use cytidine analogs in vitro ${ }^{4,5}$, which are not selective in their inhibition of any specific DNMT isoform, nor do they present any efficacy of these techniques in vivo in GI-SMC.

To further this area of research, we have selectively eliminated the methyltransferase, Dnmt1, from the murine SMC genome. Dnmt1 is known as the maintenance methyltransferase as its main enzymatic function is to faithfully restore methylation marks on newly formed daughter strands of DNA ${ }^{21}$. Deficiency of Dnmt1 caused loss of the intestinal tunica muscularis and halted peristaltic contractions which induced intestinal dilation and death around P21. CpG methylation is significantly reduced genome-wide at promoters, exons, and introns. We also found that markers of mature SMC are lost, pro-apoptotic gene expression is increased, and Dnmt1 knockout (KO) SMCs cytoplasmically accumulate a large number of coated vesicles. Furthermore, we noted consistent patterns of dysregulation of DNMT1 and TET3, an enzyme that initiates the demethylation of $\mathrm{DNA}^{22}$, between $\mathrm{KO}$ mice and variously diseased human GI tissue, including colon/anal cancers and Crohn's disease. To our knowledge, this is the first in vivo evidence showing the requirement of a DNA methyltransferase, and the importance of DNA methylation, in the primary development and differentiation of GI-SMC.

\section{Results}

Generation, and confirmation, of deletion in SMCrestricted Dnmt1 knockout mice

As global Dnmt1 ${ }^{-1-}$ mice are embryonic lethal ${ }^{23}$, it was necessary to generate a SMC-restricted $D n m t 1^{-/} \mathrm{KO}$ line.
By crossing $s m M H C$ Cre-eGFP/+, a mouse line that selectively expresses $\mathrm{Cre}$ recombinase and eGFP under the control of a Myh11 promoter ${ }^{24}$ beginning at embryonic day 12.5 , with a Dnmt1 $1^{\text {lox/lox }}$ mouse line ${ }^{25}$, we were able to successfully generate congenital smDnmt1/-;Cre-eGFP/4 (Dnmt1-KO) mice. In smMHC $C^{\mathrm{Cre}-e G F P /+}$ (Dnmt1-WT) mice, cells that express $\mathrm{Cre}$ recombinase also express $e G F P$, thus making it possible to identify Cre expression through enhanced green fluorescent protein (eGFP) fluorescence. Knockout was confirmed through genotyping with primers specific for Cre, eGFP, known loxP sites, and deletion of floxed cassette (Supplementary Figure 1). Only those mice that were positive for $\mathrm{Cre}$, both genomic loxP sites, and the deletion of the floxed cassette (Dnmt1$\mathrm{KO})$ presented the following symptoms: Dnmt1-KO mice were born similar in size and body mass to their wild-type (WT) littermates, but they have significantly less body mass by postnatal day 15 (P15) continuing through to P21 (Fig. 1a, b) when death occurs. Gross anatomical images show intestinal dilation beginning at P10 that continues to progress until P21 (Fig. 1c) when dilation is severe enough to cause intestinal ischemia or perforation of the deteriorated intestinal wall, causing septic shock. Dnmt1-KO mice also have significantly shortened GI tracts in both the small intestine (Fig. 1d) and colon (Fig. 1e) by P21.

\section{Dynamic changes of enzymes involved in DNA methylation in Dnmt1-KO mice and diseased human tissue}

DNA methylation is positively regulated by DNMT1/ DNMT3A/DNMT3B and is negatively regulated by the Ten-Eleven Translocation (TET) family of proteins (TET1/TET2/TET3). In the tunica muscularis of Dnmt1WT mice, expression levels of DNMT1 and DNMT3A are developmentally dynamic while DNMT3B is constantly expressed at all time points (Fig. 2a). DNMT1 is highly expressed at embryonic day 18 (E18) and gradually decreases from birth to P20, with the opposite pattern being seen with DNMT3A. Intriguingly, temporal expression levels of TET1/TET2/TET3 are reduced in Dnmt1-KO with the reductions of TET2 and TET3 being significant by P15. DNMT1 levels were drastically reduced in Dnmt1-KO after P1 and the protein was not detected at P20 (Fig. 2a). As previously mentioned, in Dnmt1-KO smooth muscle tissue, TET2 and TET3 levels were significantly reduced (Fig. 2d), suggesting that expression of these TET isoforms may be dependent on DNMT1. Furthermore, as Dnmt1-KO smooth muscle tissue developed, potentially compensatory DNMT3A induction occurred (Fig. 2a). Also, as DNMT1 begins to be reduced in Dnmt1-KO, so are markers of SMC maturity and contractibility, including SRF, MYH11, and SM22 $\alpha$ (Fig. 2b, d). Since MYH11 and SM22 $\alpha$ (Tagln) are SRF targets ${ }^{12}$, the reduction of SRF in Dnmt1-KO could result in, or exacerbate, the reduced expression of these SRF 
a

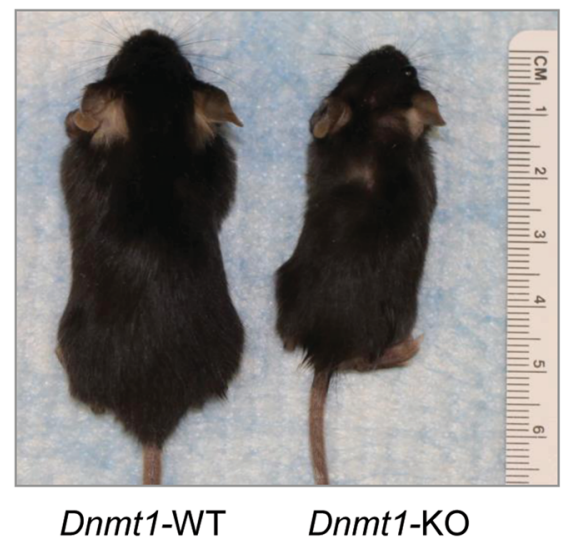

C
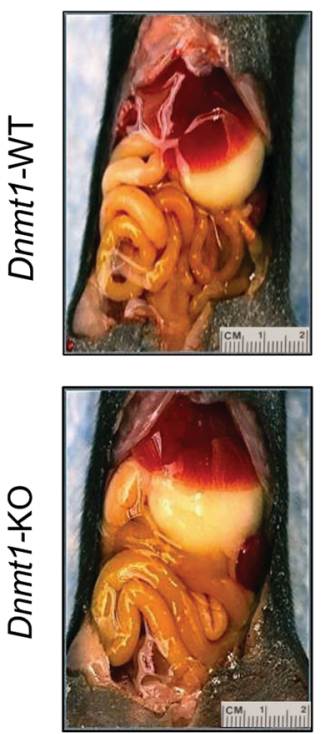

d

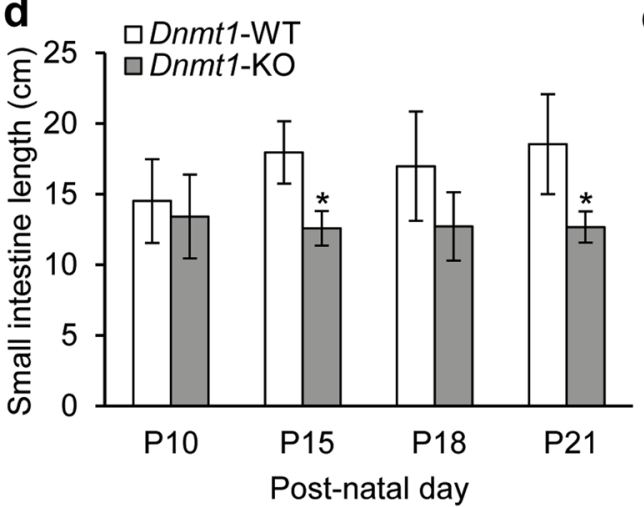

b

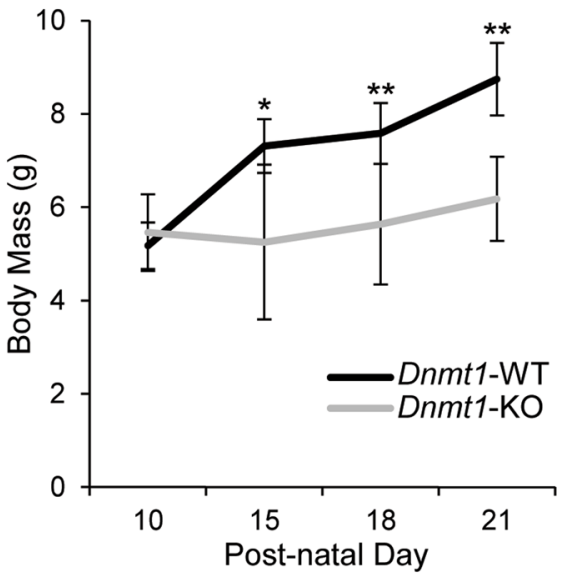

P18
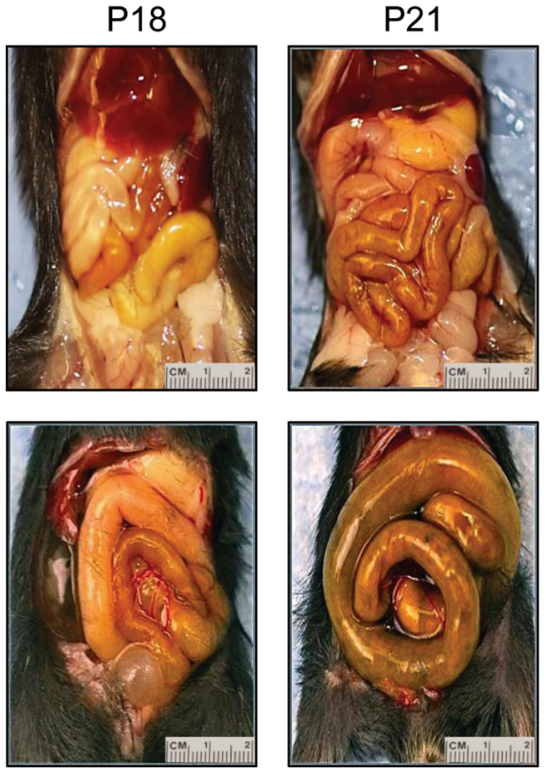

e
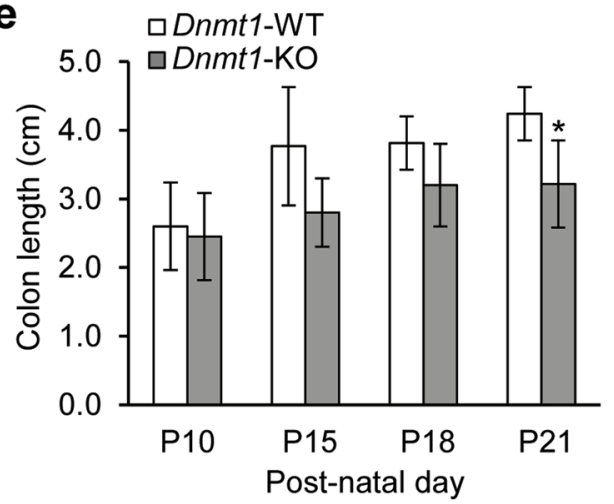

Fig. 1 SMC-restricted congenital Dnmt1-KO mice are smaller in size/mass with shorter Gl tracts that become increasingly dilated over time. a SMC-restricted congenital Dnmt1-KO mice at P2O are smaller when compared to Dnmt1-WT littermates. $\mathbf{b}$ No significant differences in body mass were seen between Dnmt1-KO mice and Dnmt1-WT mice until P15 when WT mice continued to gain mass and Dnmt1-KO began to lose mass. Dnmt1-KO slowly began to regain mass by P18 continuing into P21, most likely due to lack of peristaltic movement in the intestine causing buildup of food in the small intestine. c By P10, dilation can be seen in the small intestine of Dnmt1-KO mice with dilation increasing in size until P21 when death occurs. $\mathbf{d}$, e By P21, Dnmt1-KO mice have significantly shortened small intestines and colons. For (b, d, $\mathbf{e}) n=10$, error bars are SD; ${ }^{*} p<0.005$; ** $p<0.001$, unpaired $t$-test 


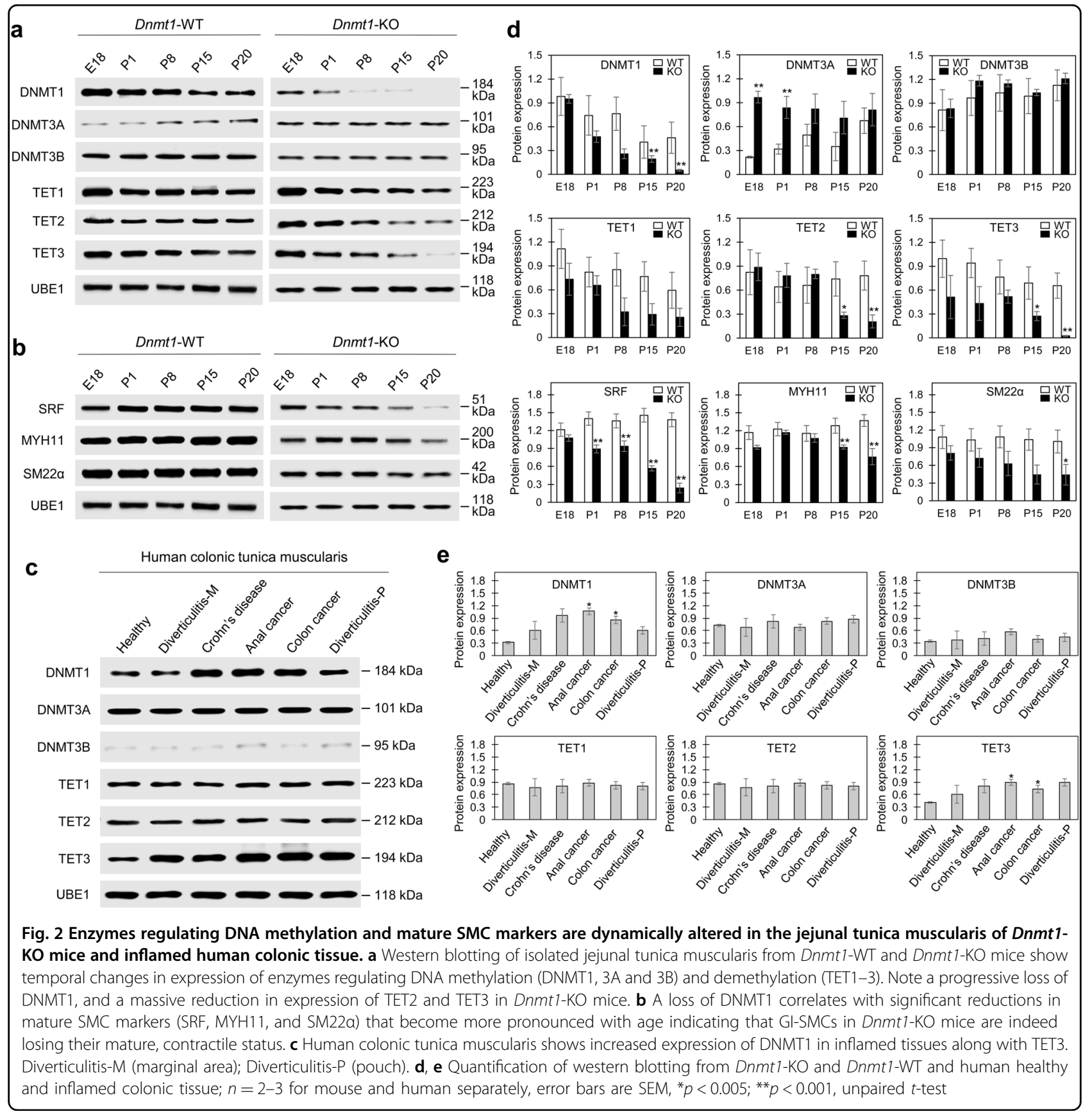

target genes and their subsequent protein products. Due to aberrant methylation patterns being identified in colorectal cancer ${ }^{26}$, we examined if DNMT1 is differentially expressed in the inflamed tunica muscularis from human patients with either Crohn's disease, colon/anal cancer, or diverticulitis. We detected increases in DNMT1 as well as increases in TET3 in the inflamed smooth muscle showing that DNMT1 can regulate TET3 across species (Fig. 2c). Quantitation of protein showed that the increases in DNMT1 and TET3 were significant in the inflamed smooth muscle from anal or colon cancer samples (Fig. 2e). Intriguingly, the correlated expression of DNMT1 and TET3 in the inflamed human tissue is consistent with the smooth muscle of Dnmt1-WT and Dnmt1-KO mice (Fig. 2a), suggesting a role for DNMT1 in regulating the expression of TET3.

\section{Dnmt1-KO mice gradually lose tunica muscularis and necessary contractile proteins and accumulate coated vesicles in SMCs}

Hematoxylin and eosin staining revealed that Dnmt1-KO mice begin to lose both muscle layers of the 

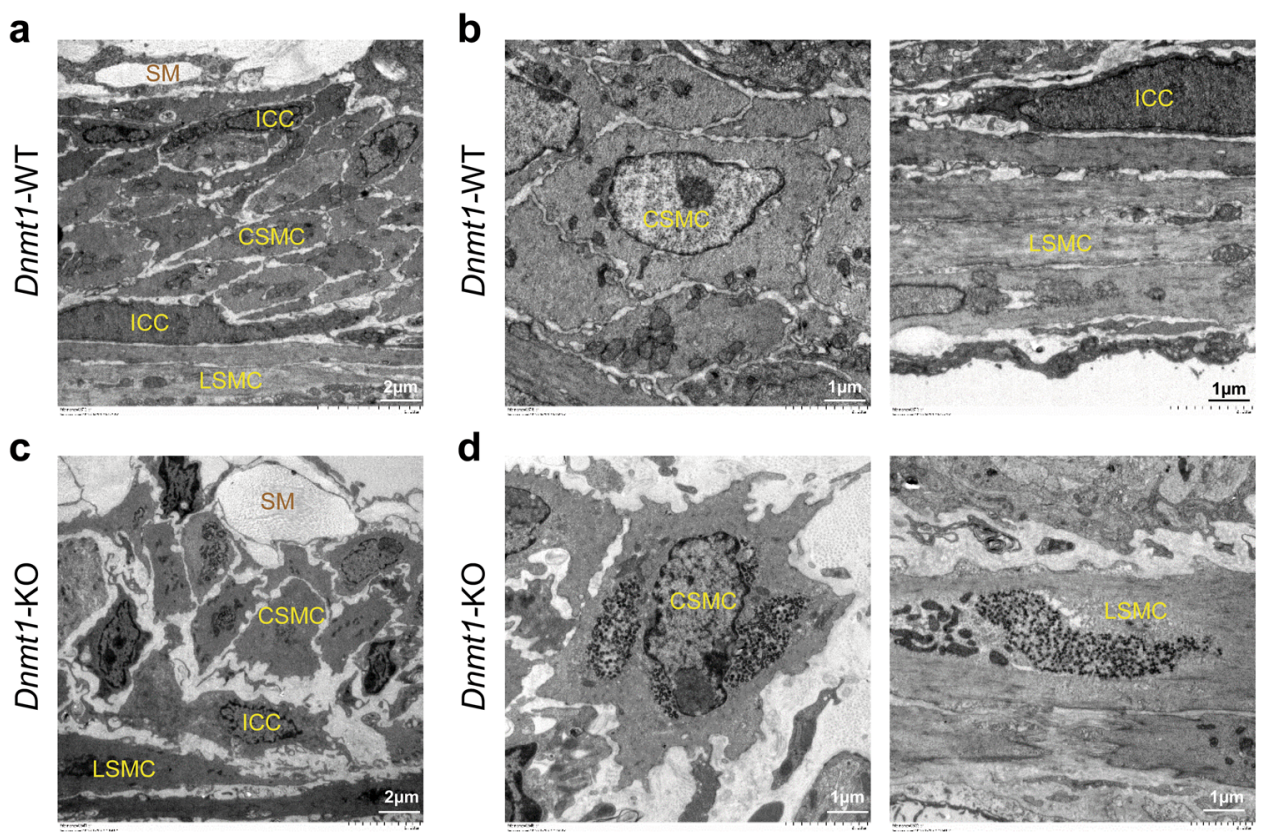

d
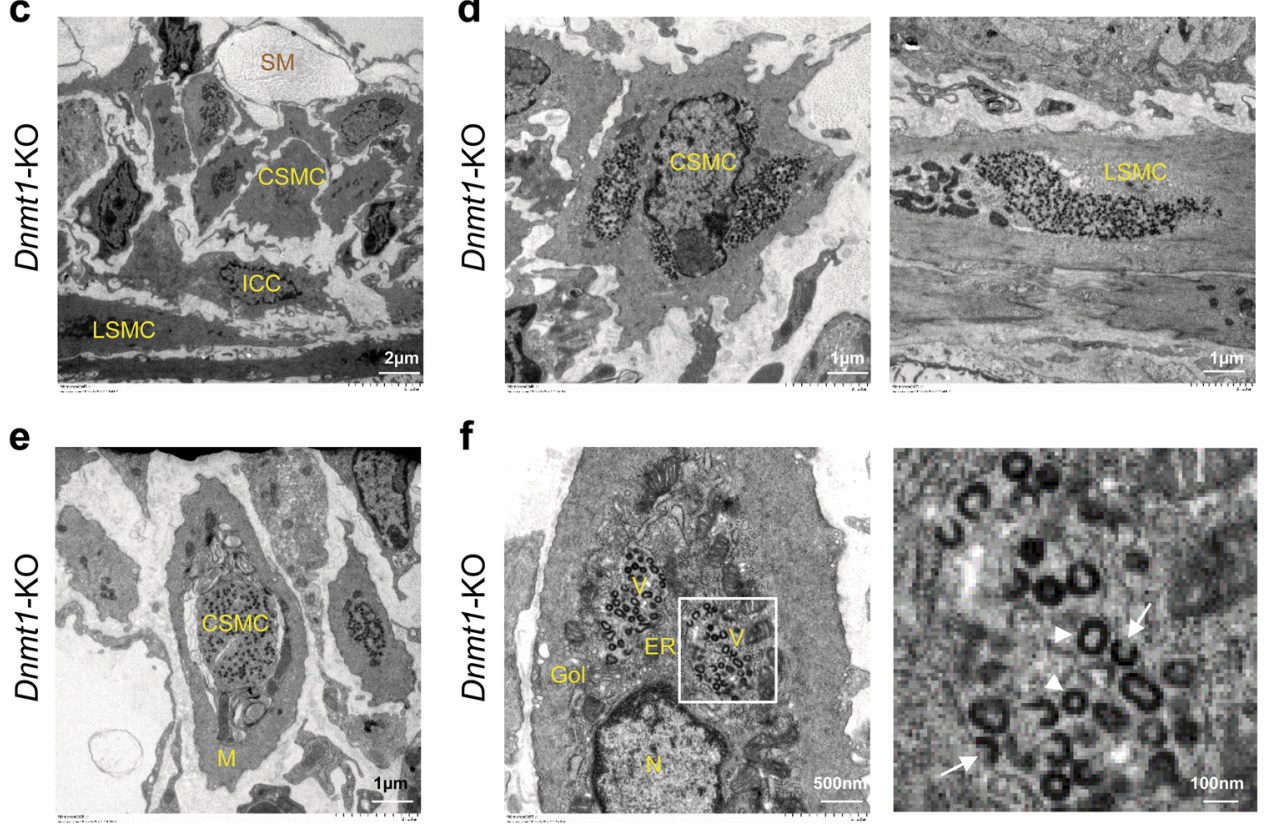

Fig. 3 Electron microscopy (EM) reveals degeneration of smooth muscle and presence of coated vesicles in Dnmt1-KO SMC. a, b EM crosssection images of P21 Dnmt1-WT jejunum. c-f EM cross-section images of P21 Dnmt1-KO jejunum. Both the circular (CM) and longitudinal muscle (LM) layers in Dnmt7-WT jejunum are intact (a, b), complete with organized nuclei in SMC as well as the presence of ICC (ICC), and submucosal cells (SM). Degenerated SMC within the tunica muscularis in the jejunum of Dnmt1-KO mice (c, $\mathbf{d})$ show cellular fragmentation, as well as a loss of nuclear organization. e A portion of a degenerated SMC with coated vesicles from a Dnmt1-KO mouse found phagocytosed in a macrophage (M). $\mathbf{f}$ Degenerated SMCs in Dnmt1-KO mice accumulate coated vesicles ( $V$ ) trapped in the vicinity of the endoplasmic reticulum (ER) and Golgi (Gol), open vesicles: arrows; closed vesicles: arrowheads

tunica muscularis at noticeable levels by P15 but did not have a significant loss of either layer until P21, with the more drastic tissue loss being found in the circular layer (Supplementary Figure 2). To confirm that the loss of tissue was indeed due to a loss of mature SMCs, immunohistochemical analysis was performed. As MYH11 is the most selective marker of mature and contractile $\mathrm{SMCs}^{27}$, antibodies targeting MYH11 were used to confirm the loss of mature SMCs alongside the endogenous eGFP reporter expression based on Myh11 expression. As expected, MYH11 levels in the tunica muscularis of Dnmt1-WT mice continued to increase from P10 to P21, while in Dnmt1-KO MYH11 levels waned, starting at P15 until there was no detectable MYH11 signal at P21, indicating a complete loss of mature SMCs (Supplementary Figure 2D). The loss of MYH11 in the SMCs of Dnmt1-KO mice results in SMCs that are no longer contractile, leading to a weakened tunica muscularis, allowing for intestinal distention. Electron microscopy of the tunica muscularis from P21 Dnmt1-KO mice revealed a loss of intercellular connections between SMCs, submucosa cells, and interstitial cells of Cajal (ICC), the necessary pacemaking cells in peristaltic contractions ${ }^{28}$ (Fig. 3c). We also noted cellular fragmentation and the loss of nuclear/nucleolar organization within Dnmt1-KO SMCs (Fig. 3c, d). Furthermore, we were able to detect macrophages engulfing the deteriorated SMCs in Dnmt1$\mathrm{KO}$ mice (Fig. 3e). Finally, we found that almost all 
a

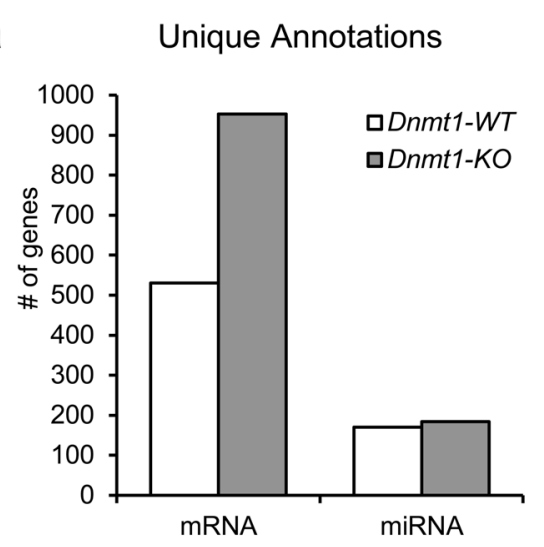

C

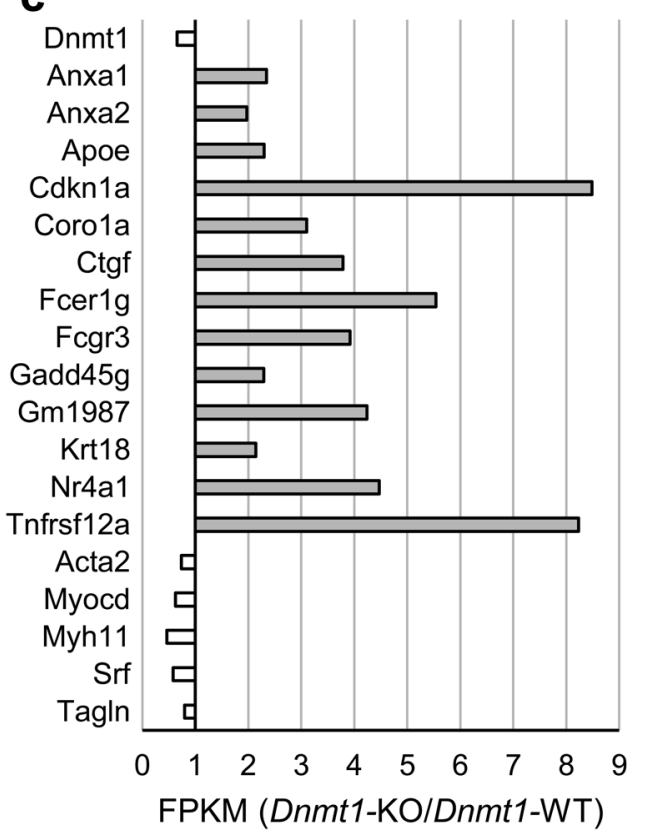

b Dnmt1-KO

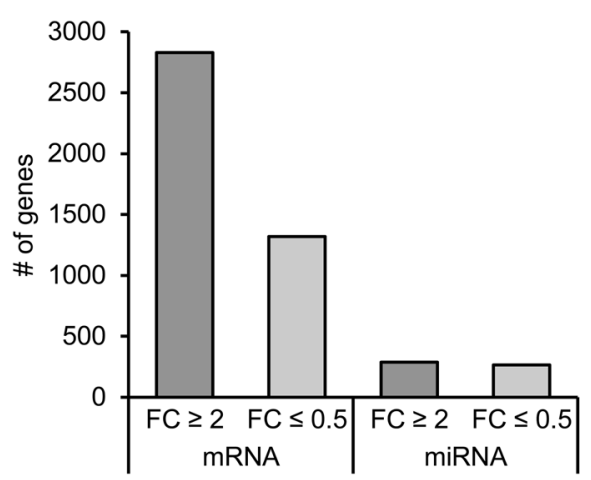

d

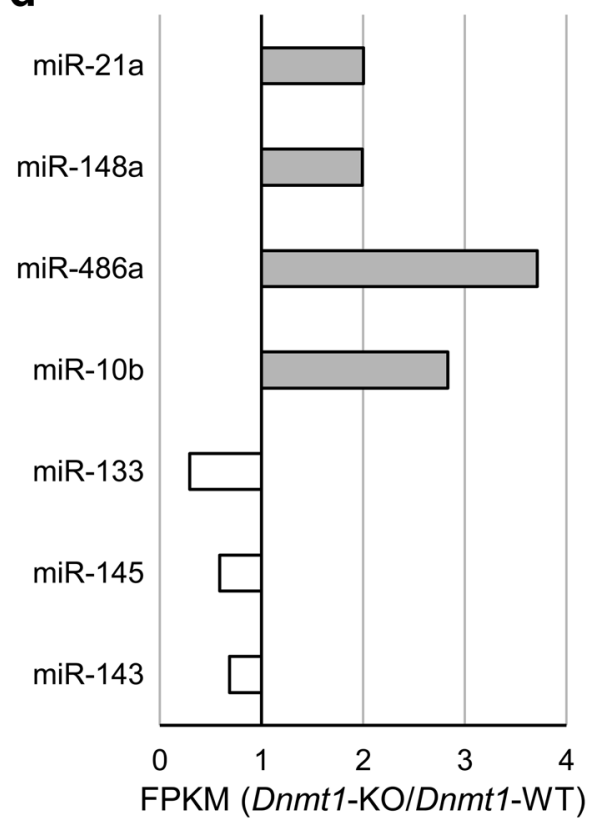

e

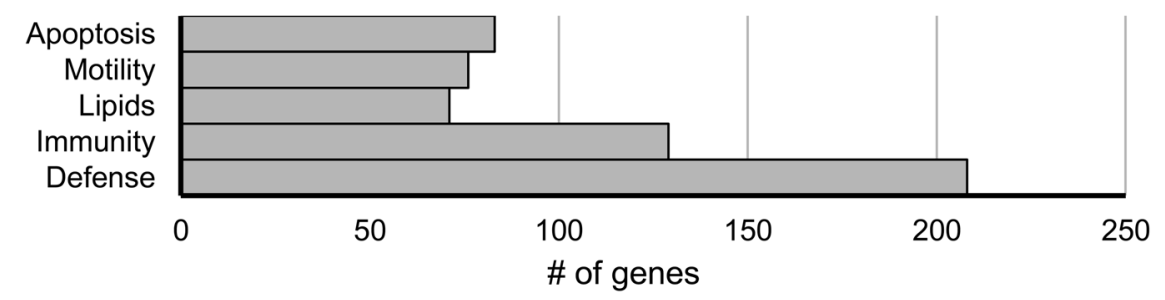

Fig. 4 Transcriptomic analysis reveals dramatic changes of expression in Dnmt1-KO jejunal tunica muscularis. a, $\mathbf{b}$ The expression profile of Dnmt1-KO jejunal tunica muscularis shows a higher number of unique mRNA transcripts and more genes that increased their expression than reduced their expression when compared to Dnmt1-WT. Changes in overall miRNA expression were not as dramatic. c Increases in several genes associated with apoptosis or lipid processing were upregulated in Dnmt1-KO mice. Additionally, transcript levels of SMC markers (Srf, Myh11) are reduced along with the SRF transcriptional co-activator, Myocd. $\mathbf{d}$ Although overall miRNA changes are not as pronounced as mRNA changes in Dnmt1-KO mice, there are select miRNAs that increase their expression level. e A gene ontology evaluation uncovered the prevalence of five general categories enriched in Dnmt7-KO jejunal tunica muscularis with many genes relating to defense, immunity, lipids, motility, and apoptosis being found

Dnmt1-KO SMCs contained small coated vesicles (Fig. 3d, f), which were not associated with Dnmt1-WT SMC (Fig. 3a, b). Dnmt1-KO SMC appeared to accrue coated vesicles in their cytosol that congregated in distinct regions around the endoplasmic reticulum and Golgi (Fig. 3f). 
a

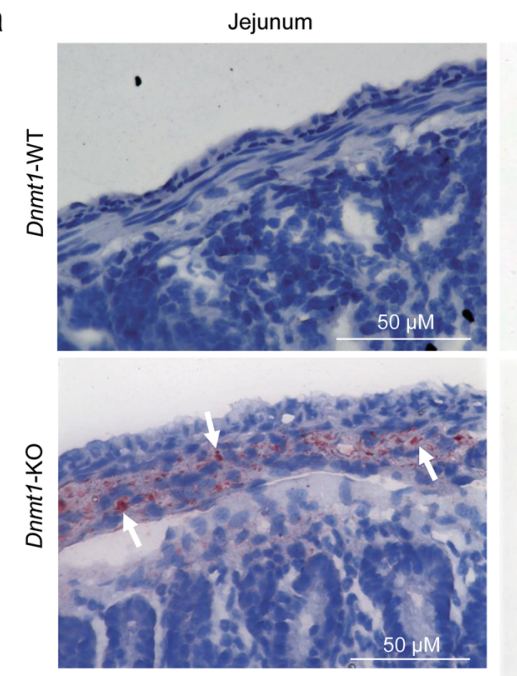

C
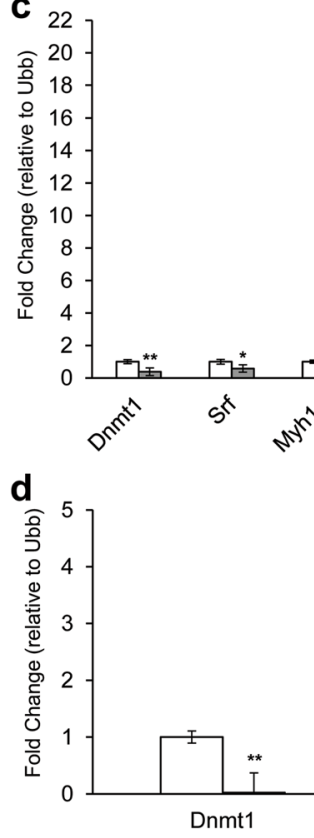

Mesentery Stain Control

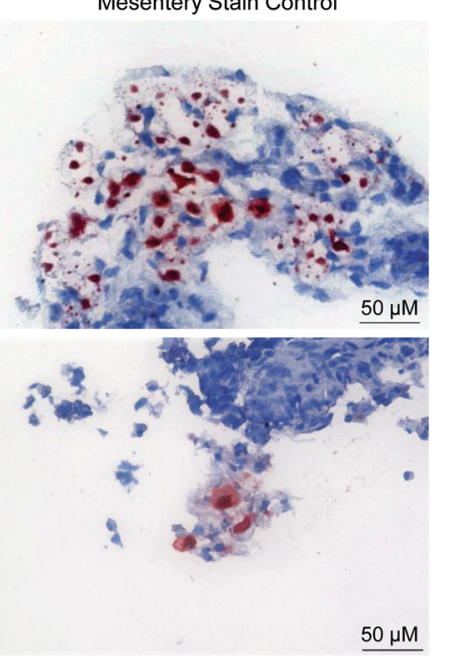

Isolated Tunica Muscularis

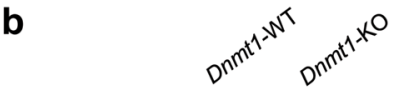

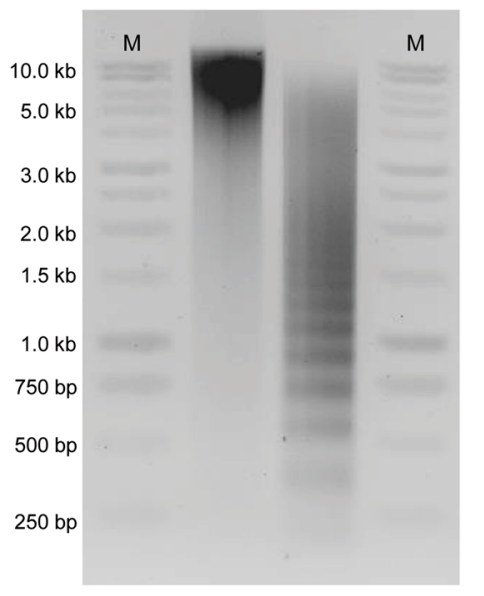

口Dnmt1-WT aDnmt1-KO
M 


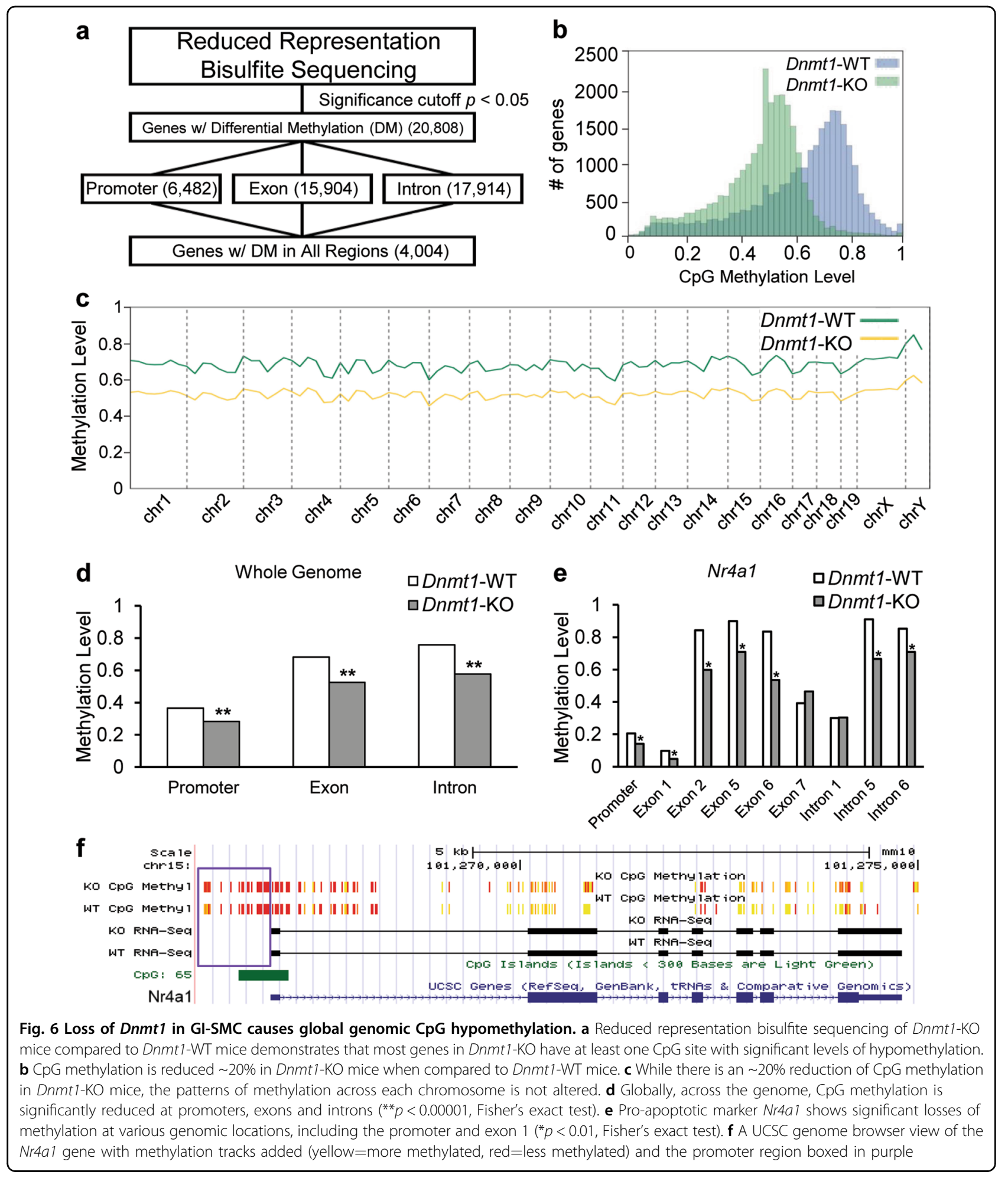

Dnmt1-WT with approximately equal amounts of unique miRNA annotations between Dnmt1-KO (184) and -WT (170) mice (Fig. 4a). When compared to Dnmt1-WT mice, Dnmt1-KO mice have 2,829 genes that at least double their expression and only 1,319 that lose at least half their expression, with similar amounts of both upregulated (289) and downregulated (265) miRNA transcripts (Fig. 4b). The differences between the relative amount and type of mRNA/miRNA transcripts expressed exposes a remodeled transcriptome in Dnmt1-KO mice. 


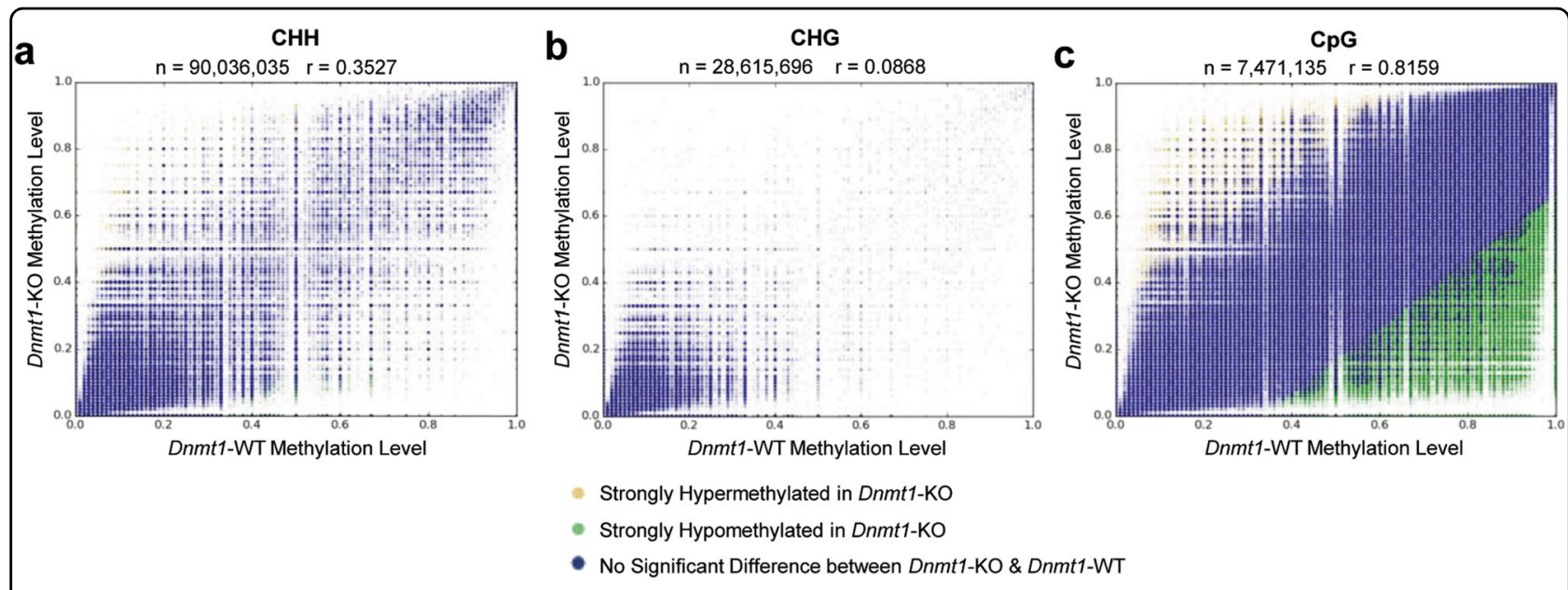

Fig. 7 Only CpG sites show large loss of methylation in Dnmt1-KO. a, b While CHH sites and CHG sites did have individual sites that changed their methylation status in Dnmt1-KO mice, there was no strong genome-wide change in methylation status. c At CpG sites in Dnmt1-KO mice, there was marked hypomethylation at multiple site across the genome which was not observed in either $\mathrm{CHH}$ or $\mathrm{CHG}$ sites. Each dot represents an individual cytosine that mapped to both Dnmt1-KO and Dnmt1-WT samples; $r$ is Pearson's correlation coefficient and $n$ is the total number of overlapping sites for each type of cytosine site

Dnmt1-KO mice have considerable losses of SMC marker transcripts necessary for mature functioning, including Acta2 (25.7\% loss), Myocd (37.4\% loss), Myh11 (53.2\% loss), Srf(41.7\% loss), and Tagln (19.9\% loss) (Fig. 4c) as well as reductions in necessary SMC miRNAs miR-143 (31.1\% loss), miR-145 (40.8\% loss), and miR-133 (70.4\% loss) (Fig. 4d). Since the transcription factors SRF and MYOCD are required for Myh11 expression ${ }^{29}$, and the previously mentioned miRNAs ${ }^{30}$, the reduction of $S r f$ and Myocd exacerbates the deterioration of SMCs as Srf expression in Dnmt1-KO does not become significantly reduced until P15 (Supplementary Figure 3) and therefore is likely not the primary insult involved in the loss of GISMCs. We also noted increases in miRNAs associated with regulating cellular identity and proliferation including, miR-10b $b^{31}$ miR-21a ${ }^{32}, \operatorname{miR}-486 a^{33}$, and miR-148a. MiR-148a is an established inhibitor of Dnmt1 expression $^{34}$, thus Dnmt1-KO SMCs, through increased miR148a, likely create even further repression of Dnmt1 expression. Furthermore, both miR-10b and miR-148a expression are known to regulated by genomic CpG methylation $^{31,35}$. For mRNA analysis, we selected 392 overexpressed genes in the tunica muscularis of Dnmt1KO mice and 365 of them had some level of significant demethylation (Supplementary Figure 4). Gene ontology (GO) examination revealed that the most overrepresented biological process categories included those associated with lipids, apoptosis, defense responses to stress/stimuli, and immune responses (Fig. 4e and Supplementary Table 4). The quantitative increase in expression levels of many of these genes that were found to be upregulated in Dnmt1-KO varied widely (Fig. 4c).
We selected upregulated gene groups associated with lipids and apoptosis for further study. Oil red $\mathrm{O}$ staining of cross-sections from Dnmt1-KO mice confirmed an accumulation of lipids in their jejunal tunica muscularis (Fig. 5a), consistent with our mRNA-sequencing and GO term analysis. Next, we examined if Dnmt1-KO SMCs were undergoing apoptosis. DNA fragmentation is a hallmark of apoptosis ${ }^{36}$ and was observed in Dnmt1-KO tunica muscularis at P21 (Fig. 5b). Our transcriptome represents the entirety of the tunica muscularis where the main population of cells are SMCs. However, immune cell infiltration into the tunica muscularis may be responsible for the increases in genes associated with immunity and other GO categories. With the use of flow cytometry, we removed cells from the tunica muscularis that were $\mathrm{CD} 45^{+}$, a known marker of immune cells ${ }^{37}$, then selected for cells that were CD $45^{-}$and eGFP ${ }^{+}$in P15 Dnmt1-KO and Dnmt1-WT. Indeed, the results of our flow cytometry identified $\sim 5$-fold increase in CD $45^{+}$cells within Dnmt1KO tissue when compared to Dnmt1-WT mice while $\mathrm{CD}^{-} 5^{-}$and $\mathrm{eGFP}^{+} \mathrm{SMC}$ were reduced $\sim 3$-fold (Supplementary Figure 5). Sorted CD45, eGFP ${ }^{+}$cells represented isolated SMCs. We confirmed that isolated SMCs from Dnmt1-KO mice had almost complete ablation of Dnmt1, significant reductions in Myh11 and Srf expression, and dramatic increases in the pro-apoptotic genes Gadd45g and Nr4a1 (Fig. 5d). However, we found other genes related to apoptosis and lipids upregulated in P15 tissue that were not upregulated in isolated Dnmt1-KO SMCs, indicating that their upregulation is likely due to immune cells infiltrating into the tunica muscularis (Fig. 5c, d). 


\section{Loss of Dnmt1 induces global genomic CpG hypomethylation}

Employing reduced representation bisulfite conversion sequencing, we discovered that $\sim 90 \%(20,808)$ of annotated genes in Dnmt1-KO mice had at least one CpG site with significant demethylation in either their promoter $(-1 \mathrm{~kb}$ of TSS), exons, or introns and $\sim 17 \%$ $(4,004)$ of genes had at least one CpG site with significant demethylation in all sites, encompassing their promoter, exons, and introns (Fig. 6a) with genomic hypomethylation being significant, on a genome-wide scale, across annotated promoter, exon, and intron $\mathrm{CpG}$ sites (Fig. 6d). None of these patterns were observed for genomic $\mathrm{CHG}$ or $\mathrm{CHH}$ ( $\mathrm{H}$ can be $\mathrm{A}, \mathrm{C}$, or $\mathrm{T}$ ) sites (Fig. 7a, b). Across the genome of Dnmt1-KO mice, there was a $\sim 20 \%$ loss of CpG methylation (Fig. 6c) with a majority of genes reducing their average CpG methylation levels from $0.8-0.6$ to $0.6-0.4$ (Fig. 6b). We also observed CpG sites that were strongly hypermethylated in Dnmt1-KO mice, but these sites were considerably outnumbered by the amount of strongly hypomethylated $\mathrm{CpG}$ sites (Fig. 7c). We created browser tracks that visualize and quantify the changes in $\mathrm{CpG}$ methylation within the University of California, Santa Cruz (UCSC) genome browser ${ }^{38}$ for any annotated genomic site. In addition, we added transcriptome level RNA-sequencing (RNA-seq) data for both mRNA and miRNA from jejunal smooth muscle in both Dnmt1-KO and Dnmt1-WT mice (Supplementary Table 3) to the browser. The browser tracks were installed at our Smooth Muscle Transcriptome Project webpage: https://med.unr.edu/physio/transcriptome, entitled "UCSC Smooth Muscle Methylome Browser." This browser provides a comprehensive reference for transcriptomic and genomic DNA methylation status at CpG sites in the jejunal smooth muscle of Dnmt1-WT and Dnmt1-KO mice. The browser can also interact with genome-level bioinformatics (e.g., ENCODE) data publically available in the UCSC Genome Browser. In Dnmt1-KO mice, Nr4a1 has significant losses of methylation at its promoter as well as exons 1 , 2, 5, and 6 and introns 5 and 6 (Fig. 6e), as shown on the browser (Fig. 6f), suggesting the expression of the pro-apoptotic Nr4a1 could be regulated by $\mathrm{CpG}$ methylation levels controlled by DNMT1, agreeing with previous findings ${ }^{39}$. The entirety of our CpG methylomic results and analysis for every annotated promoter, exon, and intron can be found in Supplementary Table 5.

\section{Discussion}

By eliminating Dnmt1 from the murine genome in a SMC-restricted model, we showed the in vivo necessity of Dnmt1 in the embryonic development of GI-SMCs.
SMC-restricted congenital Dnmt1-KO mice have shortened GI tracts, and lose their tunica muscularis by P21. Dnmt1-KO GI-SMC have significant genomic CPG demethylation, a loss of intercellular connectivity, decreases in necessary SMC transcripts (Myh11, Srf, miR133, miR-143/145), and increases in both pro-apoptotic genes (Gadd45g, Nr4a1) and miRNAs associated with changes in cellular identity (miR-21a, -148a, -186a, -10b). In fact, we found aberrant hypermethylation flanking a CArG box (CCATATAAGG: antisense) and SRF binding site found within the second intron of $\operatorname{Srf}$ in Dnmt1-KO (Supplementary Figure 6) that may have contributed to the reduction Srf expression, thus increasing the apoptotic potential of Dnmt1-KO SMC. Our results are similar to both congenital and inducible intestinal epithelial cell-specific Dnmt1-KO models. Both models show genomic hypomethylation, DNA damage, and increased apoptosis in affected Dnmt1 knockout cells $^{40,41}$. In the congenital intestinal epithelial model, only $35 \%$ of Dnmt 1 knockout mice live until P20, similar to our SMC-restricted Dnmt1-KO mice which die around P21 and none have ever survived past P24. When Dnmt1 is inducibly ablated from intestinal epithelial cells at later stages of development, the mouse does not die and sees an increase in Dnmt3b expression, which also directly compensated for the loss of DNA methylation due to the knockout of Dnmt1. This pattern of compensatory DNMT isoform induction due to loss of a separate DNMT could potentially be why we see increased DNMT3A seen during development in our Dnmt1-KO mice, as DNMT3A has been shown to be able to maintain methylation patterns in mouse embryonic stem cells lacking DNMT1 ${ }^{42}$.

While it is clear that GI-SMCs in congenital Dnmt1-KO mice are undergoing remodeling and apoptosis, it is not clear what the causative insult of these changes is, and it is more likely a combination of several pro-apoptotic influences including genomic CpG hypomethylation, epigenome modulation, lack of cellular connectivity, upregulation of pro-apoptotic genes/miRNAs, downregulation of required SMC proteins/miRNAs, exposure to lipoproteins, and chromosomal instability. As it pertains to pro-apoptotic genes upregulated in Dnmt1KO SMCs, Gadd45g is a marker of DNA damage, activator of the p38/c-Jun N-terminal kinase apoptotic pathway $^{43}$ and has been correlated with increased expression in a double knockout of DNMT1 and DNMT3B in vitro ${ }^{44}$. Nr4a1 is an orphan nuclear receptor that has been shown to be regulated by DNMT1 and the methylation level of its promoter region ${ }^{39}$. Expression of Nr4a1 is associated with an induction of apoptosis through multiple pathways ${ }^{45}$, as well as direct inhibition of the growth of SMCs ${ }^{46}$. As for genomic CpG hypomethylation, it is likely a causative feature 
involved in the transcriptomic remodeling of mRNA and miRNA expression, but probably does not account for all changes. Only about $10 \%$ of all miRNAs have been shown to change expression based on levels of DNA methylation ${ }^{47}$, denoting a secondary role of DNMT1 in regulating miRNA expression in GI-SMCs. DNMT1 interacts with up to 58 transcription factors ${ }^{48}$, and many epigenetic modifiers ${ }^{49}$, and thus it is probable that loss of Dnmt1 has downstream deleterious consequences outside of genomic hypomethylation. Furthermore, in Dnmt1-null cells, DNA mismatch repair is impeded and microsatellite instability increases $\sim 4$-fold ${ }^{50}$, indicating a pivotal, methylation-independent role of DNMT1 in DNA damage repair. While previous in vitro studies of complete Dnmt1 knockout in mouse embryonic stem cells have reported more dramatic genomic CpG methylation reductions from $\sim 75 \%$ to $\sim 18 \%{ }^{51}$ or lower at specific sites $^{52}$, cell-selective in vivo knockout of Dnmt1 in intestinal epithelial results in CpG methylation reductions from $\sim 75 \%$ to $\sim 45 \%{ }^{40,41}$, similar to our own in vivo findings (Fig. 6c).

Additionally, the observed lack of nuclear/nucleolar organization in electron microscopy images of Dnmt1-KO could be indicative of catastrophic chromosomal instability. Of the 25 genes in the CIN25 gene signature of chromosomal instability ${ }^{53}, 23$ were found to be upregulated in Dnmt1-KO smooth muscle tissue (data not shown). We were also able to show that congenital Dnmt1-KO mice have reduced levels of all TET proteins, and thus it is likely that Dnmt1-KO mice cannot properly regulate 5 -hmc or 5 -mc levels, causing dysregulation of the entire DNA methylation apparatus. We also showed that expression levels of DNMT1 and TET3 are associated and correlated within the inflamed tunica muscularis of colorectal cancer tissue as well as in the jejunal tunica muscularis of both Dnmt1-WT and Dnmt1-KO mice. Further studies are needed to elucidate the associations and interactions between DNMT1 and TET3 in inflamed and injured smooth muscle.

Most surprisingly, Dnmt1-KO SMC show an accumulation of lipids and lipid associated transcripts within their tunica muscularis. Many of the genes upregulated in our transcriptomic analysis are commonly associated with vSMC containing atherosclerotic plaques ${ }^{54}$. In vSMC, lipoproteins, based on oxidation status, have been implicated in changing the phenotypic identity of SMCs into either a proliferative or apoptotic fate ${ }^{55}$.

In conclusion, the lack of Dnmt1 in GI-SMC resulted in an inability to maintain de novo methylation patterns and the consequences of this loss become exacerbated with each round of mitotic division. As can be surmised, further research into all observed phenotypic aspects of Dnmt1-KO is necessary to understand all cellular consequences of DNMT1 deficiency.

\section{Methods}

Mice

In a C57BL/6 background, smMHC $\mathrm{Cre}^{\mathrm{CeGFP} /+}$ mice $^{24}$ were crossed with Dnmt1 $1^{\text {loxllox }}$ mice $^{25}$ to make smDnmt1 $1^{-/-; C r e-e G F P /+}$ mice. PCR confirmed genotypes (Supplementary Figure 1). Primer sequences are found in Supplementary Table 2. Mice were bred, maintained, and euthanized following guidelines set by the Institutional Animal Care and Use Committee at the University of Nevada-Reno Animal Resources.

\section{Human tissue}

Isolated human GI smooth muscle tissue sections were donated by Dr. Kent Sasse (Sasse Surgical Associates) and Dr. Laren S. Becker (Stanford University School of Medicine). Use of human tissue was approved by the Institutional Review Board at the University of Nevada, Reno.

\section{Tissue isolation and preparation}

The GI tract was extracted into 1× Hank's Calcium Free Buffer. Tissue then underwent one of two processes: (1) smooth muscle isolation and (2) whole tissue preparation for cross-sectioning. (1) The muscle layer was peeled from the mucosa for use in extractions/experiments. (2) The tissue was fixed in $4 \%$ paraformaldehyde (PFA), a dehydration in $20 \%$ sucrose/1× phosphatebuffered saline (PBS), then placed in 1:1 OCT/20\% sucrose and super-cooled by liquid nitrogen. Using a cryostat microtome (Leica, Wetzlar, Germany), $8 \mu \mathrm{M}$ thick tissue sections were cut onto slides coated with VectaBond (Maravai Biosciences, San Diego, CA, USA), and allowed to dry. Prepared slides were used for downstream immunohistochemical/histological staining.

\section{Isolation of total and small RNAs}

Isolated muscle tissue was placed in lysis buffer and homogenized by bead beating in an air-cooled Bullet Blender Storm (Next Advance, Troy, NY, USA). Total RNAs and miRNAs were isolated from smooth muscle and sorted SMCs using mirVana miRNA Isolation Kit (Ambion, Foster City, CA, USA) as previously described ${ }^{56}$. Extracted total RNAs and small RNAs were used for quantitative PCR (qPCR) and RNA-seq.

\section{Quantitative PCR}

Extracted total RNA was reverse transcribed into complementary DNA (cDNA) using SuperScript III Reverse Transcriptase (Invitrogen, Carlsbad, CA, USA), after DNA removal with DNA-free DNA Removal Kit (Ambion). cDNA samples were then quantified, diluted, and tested for quality. Standard qPCR protocol was carried out on a 7900HT Fast Real-Time PCR System (Applied Biosystems, Foster City, CA, USA) as previously 
described $^{56}$. Primer sequences are found in Supplementary Table 2 .

\section{Immunohistochemistry}

Slides were exposed to $1 \times$ Tris-buffered saline (TBS)/ $0.1 \%$ Tween 20 followed by exposure to $4 \%$ milk/ $1 \times$ TBS/ $0.1 \%$ Tween 20 . Slides were then incubated at $4{ }^{\circ} \mathrm{C}$ with $1^{\circ}$ antibody. The following day, slides were incubated with $2^{\circ}$ antibody at room temperature. After washing the slides in $1 \times$ TBS, the slides were mounted and allowed to cure. See Supplementary Table 2 for antibodies/dilutions. Slides were imaged with an Olympus FluoView FV1000 (Tokyo, Japan) confocal microscope.

\section{Hematoxylin and eosin staining}

Cross-section slides were exposed to hematoxylin, rinsed with water, and then stained in eosin Y. After several dehydrating baths of increasing ethanol (EtOH) concentration $(80 \%, 95 \%, 100 \%)$ and two terminal xylene baths, the slides were mounted and allowed to cure. Slides were imaged with an Olympus BX43 (Tokyo, Japan) brightfield microscope.

\section{Oil Red $O$ staining}

Cross-section slides were exposed to pure propylene glycol, then moved to $0.5 \%$ Oil Red $\mathrm{O}$ solution at $60^{\circ} \mathrm{C}$ followed by soaking in $85 \%$ propylene glycol. slides were then rinsed and counterstained with hematoxylin, rinsed with water, then mounted with aqueous mounting reagent and allowed to cure. Slides were imaged with a Keyence BZ-x710 (Osaka, Japan) brightfield microscope.

\section{Electron microscopy}

Whole jejunum and colon tissue were fixed (3\% glutaraldehyde and $4 \%$ PFA in $0.1 \mathrm{M}$ phosphate buffer) for several days at room temperature. Specimens were then fixed in $1 \% \mathrm{OsO}_{4}$ at $4{ }^{\circ} \mathrm{C}$, rinsed in distilled water, then block stained with saturated aqueous uranyl acetate solution, followed by dehydration in a graded series of $\mathrm{EtOH}$ and finally embedded in EPON 812. Ultrathin sections were stained with uranyl acetate and lead citrate and then examined using a Hitachi H-7650 (Tokyo, Japan) microscope.

\section{Western blot}

Isolated SM tissue was ground by mortar and pestle in modified RIPA buffer. Transferred polyvinylidene fluoride membranes were pre-blocked in $5 \%$ milk, then exposed to $1^{\circ}$ antibody at $4{ }^{\circ} \mathrm{C}$ in $2 \%$ milk/0.05\% Tween 20 at varying dilutions. The membranes were exposed to $2^{\circ}$ antibody at room temperature. All antibodies used can be found in Supplementary Table 2. Imaging by UVP EC3 (Upland, CA, USA) Imaging system. Quantification of western blot banding patterns was done using ImageJ and histograms were produced in SigmaPlot (Systat, San Jose, CA, USA).

\section{DNA methylome}

Genomic DNA was isolated from jejunal tunica muscularis at P15 (2 males and 2 females for both Dnmt1-KO and Dnmt1-WT; $n=4$ ) using Qiagen (Hilden, Germany) AllPrep DNA spin columns. DNA samples were pooled and shipped to Zymo Research Corporation (Irvine, CA, USA) where the samples underwent Methyl-MidiSeq which involves genomic DNA extraction, fragmentation, end modification, bisulfite conversion, limited amplification, and sequencing. After sequencing, bioinformatic processing was utilized in comparing amount of methylation at CpG, $\mathrm{CHH}$, and CHG sites between Dnmt1-WT and Dnmt1-KO samples. Methylation ratios were calculated (methylated cytosines/total cytosine reads) for individual sites and then averaged across regions (exons, introns, promoters $=-1 \mathrm{~kb}$ of TSS). These averages were then used in Fisher's exact test to quantify significant changes $(p<0.05)$ in methylation. The reference genome used was GRCm38/mm10. The data from our bisulfite conversion sequencing were submitted to the Gene Expression Omnibus (GEO) repository with the following accession numbers: GSM2947783, Dnmt1-WT jejunum; GSM2947784, Dnmt1-KO jejunum.

\section{RNA sequencing}

Total RNAs and small RNAs isolated from Dnmt1-KO and Dnmt1-WT jejunal tunica muscularis at P15 (2 males and 2 females for both Dnmt1-KO and Dnmt1-WT; $n=$ 4) were shipped to LC Sciences (Houston, TX, USA) where mRNA-seq and miRNA-seq were performed. Sequencing data analysis, alignments, annotations, and bioinformatics processing were carried out as previously described $^{57}$. The reference genome used was GRCm38/ mm10. Our RNA-seq data for both mRNA and miRNA were submitted to the GEO repository with the following accession numbers: GSM2936348, Dnmt1-WT jejunum mRNA; GSM2936349, Dnmt1-KO jejunum mRNA; GSM2936350, Dnmt1-WT jejunum miRNA; GSM2936351 Dnmt1-KO jejunum miRNA.

\section{Cell sorting}

Cells were dispersed as previously described ${ }^{58}$. Cells were incubated with APC-Cy7 Anti-CD45 (Biolegend, Clone 30-F11, $1.0 \mu \mathrm{g} / \mathrm{mL}$; San Diego, CA, USA) followed by washing with $\mathrm{PBS} / 1 \%$ FBS. Resuspended cells had Hoechst $33258(1 \mu \mathrm{g} / \mathrm{mL})$ added as a viability marker. Cells were sorted and analyzed using the BD Biosciences (San Jose, CA, USA) FACSAria II Special Order Research Product with a $130 \mu \mathrm{m}$ nozzle with sheath pressure at 12 psi. The $355 \mathrm{~nm}$ laser excited Hoechst 33258 with a 450/ 
$50 \mathrm{~nm}$ bandpass filter. The eGFP was excited using a 488 $\mathrm{nm}$ laser with a 530/30 $\mathrm{nm}$ bandpass filter. A neutral density filter 2 was used on the forward scatter detector due to the high forward scatter properties. Cells that were $\mathrm{CD}_{4} 5^{-}$and $\mathrm{eGFP}^{+}$were sorted into $\mathrm{PBS} / 1 \% \mathrm{FBS}$. Acquisition was performed on BD FACSDiva 8.0 and TreeStar Flowjo (Ashland, OR, USA) was used to generate figures.

\section{Acknowledgements}

We would like to thank Drs. Michael Kotlikoff and Laurie Jackson-Grusby for their generous donations of the congenital smMHC $C^{\text {ree-GFP/+ }}$ and Dnmt $1^{\text {lox/lox }}$ mouse lines respectively as well as Benjamin J. Weigler and Walt F. Mandeville (Laboratory Animal Medicine, University of Nevada, Reno) for the excellent animal services provided to the mice. We would also like to thank Dr. Kent M. Sanders for COBRE Core B and Byoung H. Koh for the cell sorting services as well as Dr. Tong Zhou for his assistance in bioinformatic data presentation. B.G. J. was supported by an award from the J. M. Hitchcock, Graduate Student Research Fund.

This study was supported by National Institutes of Health (NIH) (DK094886 and DK103055 to S.R.) and COBRE Phase III FACSAria (P30 GM1 10767 to Kent M. Sanders). The funders had no role in study design, data collection/analysis, decision to publish, or preparation of the manuscript.

\section{Author details}

'Department of Physiology and Cell Biology, University of Nevada School of Medicine, Reno, NV 89557, USA. 'Department of Morphological and Physiological Sciences, University of Fukui, Fukui 910-8507, Japan. ${ }^{3}$ Sasse Surgical Associates, Reno, NV 89502, USA. ${ }^{4}$ Gastroenterology and Hepatology, Stanford University School of Medicine, Stanford, CA 94305, USA

\section{Authors' contributions}

R.M.B., B.G.J., S.E.H., and S.R. designed research; R.M.B., B.G.J., S.E.H., and K.H. performed research; B.G.J., R.M.B., S.E.H., and S.R. analyzed data; K.C.S. and L.S.B. contributed materials. B.G.J. wrote the paper and S.R revised it.

\section{Conflict of interest}

The authors declare that they have no conflict of interest.

\section{Publisher's note}

Springer Nature remains neutral with regard to jurisdictional claims in published maps and institutional affiliations.

\section{Funding}

Wessex Cancer Trust, CRUK—Southampton Internal Pilot Grant, EU-FP7 Marie Curie (CANOMICS), Annual Adventures in Research-University of Southampton, EU-Excellence II-Systems Biology Framework FRA-SYS (Grant 4072), Cancer Research UK (Grants C34999/A13719 and RG84119), and MRC Clinician Scientist Fellowship (Grant G1002565).

Supplementary Information accompanies this paper at https://doi.org/ 10.1038/s41419-018-0495-z.

Received: 30 October 2017 Revised: 31 January 2018 Accepted: 12 February 2018

Published online: 27 April 2018

\section{References}

1. Wedel, T. et al. Novel smooth muscle markers reveal abnormalities of the intestinal musculature in severe colorectal motility disorders. Neurogastroenterol. Motil. 18, 526-538 (2006).

2. Rolle, U., O'Briain, S., Pearl, R. H. \& Puri, P. Megacystis-microcolon-intestinal hypoperistalsis syndrome: evidence of intestinal myopathy. Pediatr. Surg. Int. 18, 2-5 (2002).
3. Liu, R., Leslie, K. L. \& Martin, K. A. Epigenetic regulation of smooth muscle cell plasticity. Biochim. Biophys. Acta 1849, 448-453 (2015).

4. Hu, B., Gharaee-Kermani, M., Wu, Z. \& Phan, S. H. Epigenetic regulation of myofibroblast differentiation by DNA methylation. Am. J. Pathol. 177, 21-28 (2010).

5. Ning, $Y$. et al. 5-Aza-2'-deoxycytidine inhibited PDGF-induced rat airway smooth muscle cell phenotypic switching. Arch. Toxicol. 87, 871-881 (2013).

6. Hinoue, T. et al. Genome-scale analysis of aberrant DNA methylation in colorectal cancer. Genome Res. 22, 271-282 (2012).

7. Sadler, T. et al. Genome-wide analysis of DNA methylation and gene expression defines molecular characteristics of Crohn's disease-associated fibrosis. Clin. Epigenetics 8, 30 (2016).

8. Ramchandani, S., Bhattacharya, S. K., Cervoni, N. \& Szyf, M. DNA methylation is a reversible biological signal. Proc. Natl. Acad. Sci. USA 96, 6107-6112 (1999).

9. Gardiner-Garden, M. \& Frommer, M. CpG Islands in vertebrate genomes. J. Mol. Biol. 196, 261-282 (1987).

10. Jones, P. A. Functions of DNA methylation: islands, start sites, gene bodies and beyond. Nat. Rev. Genet. 13, 484-492 (2012).

11. McDonald, O. G. \& Owens, G. K. Programming smooth muscle plasticity with chromatin dynamics. Circ. Res. 100, 1428-1441 (2007).

12. Miano, J. M., Long, X. \& Fujiwara, K. Serum response factor: master regulator of the actin cytoskeleton and contractile apparatus. Am. J. Physiol. Cell Physiol. 292, C70-C81 (2007).

13. Miano, J. M., Cserjesi, P., Ligon, K. L., Periasamy, M. \& Olson, E. N. Smooth muscle myosin heavy chain exclusively marks the smooth muscle lineage during mouse embryogenesis. Circ. Res 75, 803-812 (1994).

14. Skalli, O. et al. A monoclonal antibody against a-smooth muscle actin: a new probe for smooth muscle differentiation. J. Cell Biol. 103, 2787-2796 (1986).

15. Torella, D. et al. MicroRNA-133 controls vascular smooth muscle cell phenotypic switch in vitro and vascular remodeling in vivo. Circ. Res. 109, 880-893 (2011).

16. Cordes, K. R. et al. MiR-145 and miR-143 regulate smooth muscle cell fate and plasticity. Nature 460, 705-710 (2009).

17. Wu, W., Shen, X. \& Tao, S. Characteristics of the CArG-SRF binding context in mammalian genomes. Mamm. Genome 21, 104-113 (2010).

18. McDonald, O. G., Wamhoff, B. R., Hoofnagle, M. H. \& Owens, G. K. Control of SRF binding to CArG box chromatin regulates smooth muscle gene expression in vivo. J. Clin. Invest. 116, 36-48 (2006).

19. Warnecke, P. M. \& Clark, S. J. DNA methylation profile of the mouse skeletal aactin promoter during development and differentiation. Mol. Cell Biol. 19, 164-172 (1999)

20. Yamamura, H. et al. Structure and expression of the human SM22a gene, assignment of the gene to chromosome 11, and repression of the promoter activity by cytosine DNA methylation. J. Biochem. 122, 157-167 (1997).

21. Robert, M. F. et al. DNMT1 is required to maintain $\mathrm{CpG}$ methylation and aberrant gene silencing in human cancer cells. Nat. Genet 33, 61-65 (2003).

22. Pastor, W. A., Aravind, L. \& Rao, A. TETonic shift: biological roles of TET proteins in DNA demethylation and transcription. Nat. Rev. Mol. Cell Biol. 14, 341-356 (2013).

23. Li, E., Bestor, T. H. \& Jaenisch, R. Targeted mutation of the DNA methyltransferase gene results in embryonic lethality. Cell 69, 915-926 (1992).

24. Xin, H.-B., Deng, K.-Y., Rishniw, M., Ji, G. \& Kotlikoff, M. I. Smooth muscle expression of Cre recombinase and eGFP in transgenic mice. Physiol. Genom. 10, 211-215 (2002).

25. Jackson-Grusby, L. et al. Loss of genomic methylation causes p53-dependent apoptosis and epigenetic deregulation. Nat. Genet. 27, 31-39 (2001).

26. Coppedè, F. Epigenetic biomarkers of colorectal cancer: focus on DNA methylation. Cancer Lett. 342, 238-247 (2014).

27. Owens, G. K., Kumar, M. S. \& Wamhoff, B. R. Molecular regulation of vascular smooth muscle cell differentiation in development and disease. Physiol. Rev. 84, 767-801 (2004)

28. Sanders, K. M., Ördög, T., Koh, S. D., Torihashi, S. \& Ward, S. M. Development and plasticity of interstitial cells of Cajal. Neurogastroenterol. Motil. 11, 311-338 (1999).

29. Wang, Z., Wang, D.-Z., Teg Pipes, G. C. \& Olson, E. N. Myocardin is a master regulator of smooth muscle gene expression. Proc. Natl. Acad. Sci. USA 100, 7129-7134 (2003).

30. Park, C. et al. Serum response factor-dependent microRNAs regulate gastrointestinal smooth muscle cell phenotypes. Gastroenterology 141, 164-175 (2011). 
31. Li, Z. et al. DNA methylation downregulated mir-10b acts as a tumor suppressor in gastric cancer. Gastric Cancer 18, 43-54 (2015).

32. Ji, R. et al. MicroRNA expression signature and antisense-mediated depletion reveal an essential role of microRNA in vascular neointimal lesion formation. Circ. Res. 100, 1579-1588 (2007).

33. Oh, H.K. et al. Genomic loss of miR-486 regulates tumor progression and the OLFM4 antiapoptotic factor in gastric cancer. Clin. Cancer Res. 17, 2657-2667 (2011).

34. Nan, W. et al. MicroRNA-21 and microRNA-148a contribute to DNA hypomethylation in lupus CD4+T cells by directly and indirectly targeting DNA methyltransferase 1. J. Immunol. 184, 6773-6781 (2010).

35. Lujambio, A. et al. A microRNA DNA methylation signature for human cancer metastasis. Proc. Natl. Acad. Sci. USA 105, 13556-13561 (2008).

36. Bortner, C. D., Oldenburg, N. B. E. \& Cidlowski, J. A. The role of DNA fragmentation in apoptosis. Trends Cell Biol. 5, 21-26 (1995).

37. Donovan, J. A. \& Koretzky, G. A. CD45 and the immune response. J. Am. Soc. Nephrol. 4, 976-985 (1993).

38. Kent, W. J. et al. The Human Genome Browser at UCSC. Genome Res. 12 996-1006 (2002)

39. Chen, Y.-T., Liao, J.-W., Tsai, Y.-C. \& Tsai, F.-J. Inhibition of DNA methyltransferase 1 increases nuclear receptor subfamily 4 group A member 1 expression and decreases blood glucose in type 2 diabetes. Oncotarget 7, 39162-39170 (2016).

40. Elliott, E. N., Sheaffer, K. L. \& Kaestner, K. H. The 'de novo' DNA methyltransferase Dnmt3b compensates the Dnmt1-deficient intestinal epithelium. Elife 5, 1-15 (2016).

41. Elliott, E. N., Sheaffer, K. L., Schug, J., Stappenbeck, T. S. \& Kaestner, K. H. Dnmt1 is essential to maintain progenitors in the perinatal intestinal epithelium. Development 142, 2163-2172 (2015).

42. Liang, G. et al. Cooperativity between DNA methyltransferases in the maintenance methylation of repetitive elements. Mol. Cell Biol. 22, 480-491 (2002).

43. Takekawa, M. \& Saito, H. A family of stress-inducible GADD45-like proteins mediate activation of the stress-responsive MTK1/MEKK4 MAPKKK. Cell 95, 521-530 (1998).

44. Ying, J. et al. The stress-responsive gene GADD45G is a functional tumor suppressor, with its response to environmental stresses frequently disrupted epigenetically in multiple tumors. Clin. Cancer Res. 11, 6442-6449 (2005).
45. Li, Q.-X., Ke, N., Sundaram, R. \& Wong-Staal, F. NR4A1, 2, 3 - an orphan nuclear hormone receptor family involved in cell apoptosis and carcinogenesis. Histol. Histopathol. 21, 533-540 (2006).

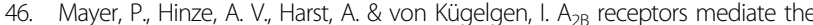
induction of early genes and inhibition of arterial smooth muscle cell proliferation via Epac. Cardiovasc. Res. 90, 148-156 (2011).

47. Han, L., Witmer, P. D., Casey, E., Valle, D. \& Sukumar, S. DNA methylation regulates microRNA expression. Cancer Biol. Ther. 6, 1284-1288 (2007).

48. Hervouet, E., Vallette, F. M. \& Cartron, P.-F. Dnmt1/transcription factor interactions: an alternative mechanism of DNA methylation inheritance. Genes Cancer 1, 434-443 (2010).

49. Qin, W., Leonhardt, H. \& Pichler, G. Regulation of DNA methyltransferase 1 by interactions and modifications. Nucleus 2, 392-402 (2011).

50. Jin, B. \& RobertsonK. D. DNA methyltransferases (DNMTs), DNA damage repair and cancer. Adv. Exp. Med. Biol. 754, 3-29 (2013).

51. Li, Z. et al. Distinct roles of DNMT1-dependent and DNMT1-independent methylation patterns in the genome of mouse embryonic stem cells. Genome Biol. 16, 115 (2015).

52. Martos, S. N. et al. Two approaches reveal a new paradigm of 'switchable or genetics-influenced allele-specific DNA methylation' with potential in human disease. Cell Discov. 3, 17038 (2017).

53. Carter, S. L., Eklund, A. C., Kohane, I. S., Harris, L. N. \& Szallasi, Z. A signature of chromosomal instability inferred from gene expression profiles predicts clinical outcome in multiple human cancers. Nat. Genet. 38, 1043-1048 (2006).

54. Lusis, A. J., Fogelman, A. M. \& Fonarow, G. C. Genetic basis of atherosclerosis: part I. New genes and pathways. Circulation 110, 1868-1873 (2004).

55. Johnson, J. L. Emerging regulators of vascular smooth muscle cell function in the development and progression of atherosclerosis. Cardiovasc. Res. 103 452-460 (2014).

56. Ro, S. et al. Cloning and expression profiling of small RNAs expressed in the mouse ovary. RNA 13, 2366-2380 (2007).

57. Lee, M. Y. et al. Smooth muscle cell genome browser: enabling the identification of novel serum response factor target genes. PLoS One 10, e0133751 (2015).

58. Ro, S. et al. A model to study the phenotypic changes of interstitial cells of Cajal in gastrointestinal diseases. Gastroenterology 138, 1068-1078 (2010). 